\title{
Cross-identification of stellar catalogs with multiple stars: Complexity and Resolution
}

\author{
Daniel Severín ${ }^{1,2}$ \\ Depto. de Matemática (FCEIA), Universidad Nacional de Rosario, Argentina \\ CONICET, Argentina
}

\begin{abstract}
In this work, I present an optimization problem which consists of assigning entries of a stellar catalog to multiple entries of another stellar catalog such that the probability of such assignment is maximum. I show a way of modeling it as a Maximum Weighted Stable Set Problem which is further used to solve a real astronomical instance and I partially characterize the forbidden subgraphs of the resulting family of graphs given by that reduction. Finally, I prove that the problem is $\mathcal{N} \mathcal{P}$-Hard.
\end{abstract}

Keywords: Cross-identification, Complexity, Maximum Weighted Stable Set Problem, Forbidden subgraphs.

\section{Introduction}

In the science of astronomy, it is common to record the position and other physical quantities of stellar objects in astronomical catalogs. They are of extreme importance for various disciplines, such as navigation, space research and geodesy. Naturally, in star catalogs, a single star has different designations

1 Partially supported by grants PICT-2016-0410 (ANPCyT) and PID ING538 (UNR).

2 Email: daniel@fceia.unr.edu.ar 
according to the catalog being used that uniquely identifies it. Suppose that $A$ and $B$ are star catalogs, and $i d_{A}, i d_{B}$ are the designations of the same star in $A$ and $B$ respectively. It is often necessary to know $i d_{B}$ given $i d_{A}$. This kind of cross-identification can be performed by software tools available on Internet, such as Xmatch ${ }^{3}$ or the web-based CDS X-Match Service ${ }^{4}$, which usually use heuristic algorithms. It was not until recently, however, that exact approaches began to be proposed. For instance, in [1], a cross-identification problem is solved through assignment problems via the Hungarian Algorithm.

The correspondence between two catalogs does not need to be one-to-one. Some stars appearing as single ones in one catalog could correspond to multiple stars in the other. Although some catalogs, such as SAO and PPM, inform whether a certain star is double or not, available cross-matching tools do not take into account this piece of information about the star.

Consider the following cross-identification problem. Given two catalogs $A$ and $B$ covering the same region of the sky and being $B$ denser than $A$, the problem consists of finding the "most probable" assignment such that every star $a$ is assigned up to $k_{a}$ stars of $B$, where $k_{a}$ is the multiplicity of $a$ informed by catalog $A$.

The original motivation to study this novel matching problem has arisen during a joint collaboration with astrophysicist Diego Sevilla [2] and whose objective has been the development of a new digital version of the Cordoba Durchmusterung, a star catalog widely used in the twentieth century.

In this work, I describe an optimization problem which I call $\mathscr{K}$-Matching Problem and I give a polynomial-time reduction to the Maximum Weighted Stable Set Problem (MWSSP). This reduction is further used for solving a real instance. I also present an open question concerning the forbidden subgraphs of the family of graphs that arise in that reduction and I identify two of the forbidden subgraphs. Then, I prove that the $\mathscr{K}$-Matching Problem is $\mathcal{N} \mathcal{P}$ Hard for a given $\mathscr{K} \geq 2$.

\section{Problem description and resolution}

Consider two star catalogs where each star is represented as elements of a set $A$ or $B$. Let $n_{A}$ and $n_{B}$ be the cardinality of $A$ and $B$ respectively.

For a given entry $a \in A$, let $k_{a}$ be the multiplicity of $a$ in the first catalog. That is, if $a$ represents a single star then $k_{a}=1$, if $a$ represents a double one

3 http://matthiaslee.github.io/Xmatch

4 http://cdsxmatch.u-strasbg.fr/xmatch 
then $k_{a}=2$, and so on. Also, let $\mathscr{K}$ be the largest multiplicity.

The resolution of our problem is divided in two phases:

- Phase 1: From the astrometric and photometric data available from catalogs, generate an instance of the $\mathscr{K}$-Matching Problem.

- Phase 2: Reduce that instance to an instance of the MWSSP and solve it.

The first phase depends on the structure of both catalogs and involves criteria in the field of Astronomy, which can be separated from the mathematical description of the problem. For that reason, it will be discussed in an Online Appendix ${ }^{5}$. In this section, only the second phase is addressed.

During the first phase, candidates sets of stars $P_{a} \subset \mathscr{P}(B)$ are generated for each $a \in A$. For instance, the set $P_{a}=\left\{\emptyset,\left\{b_{1}\right\},\left\{b_{2}\right\},\left\{b_{1}, b_{3}\right\}\right\}$ indicates that $a$ can be assigned to $b_{1}, b_{2}$, the pair $\left\{b_{1}, b_{3}\right\}$ or no one (indicated by the presence of $\emptyset$ ) with positive probability. Naturally, every $j \in P_{a}$ must satisfy $|j| \leq k_{a}$. For a given star $a \in A$ and a set $j \in P_{a}$, denote the event that " $a$ corresponds to $j$ " by $a \rightarrow j$ and its probability by $p(a \rightarrow j)$, which is computed during the first phase. Also, $\sum_{j \in P_{a}} p(a \rightarrow j)=1$.

An assignment $f: A \rightarrow \mathscr{P}(B)$ is valid when it satisfies $f(a) \in P_{a}$ for all $a \in A$, and for any $a_{1}, a_{2} \in A$ such that $a_{1} \neq a_{2}$, then $f\left(a_{1}\right) \cap f\left(a_{2}\right)=\emptyset$, i.e. candidates of $B$ assigned to $a_{1}$ and $a_{2}$ must not share common stars. Let $\mathcal{F}$ be the space of valid assignments. Each $f \in \mathcal{F}$ has a corresponding probability $p(f)=p\left(a_{1} \rightarrow f\left(a_{1}\right), a_{2} \rightarrow f\left(a_{2}\right), \ldots\right)$. We are interested in finding the most probable assignment: $f^{*} \in \operatorname{argmax}_{f \in \mathcal{F}} p(f)$. Since the number of assignments is exponential, it makes little sense to perform the computation of the real probability of each one. Thus, let us make a simplification at this point by supposing the following assumption:

for all $f \in \mathcal{F}$ and $a, a^{\prime} \in A$ such that $a \neq a^{\prime}$, events $a \rightarrow f(a)$ and $a^{\prime} \rightarrow f\left(a^{\prime}\right)$ are independent each other.

Let $\bar{p}(f)=\prod_{a \in A} p(a \rightarrow f(a))$. If the previous assumption holds, we would have $\bar{p}(f)=p(f)$. Although it usually does not hold, the assignment $f$ that maximizes $\bar{p}(f)$ is enough good for practical purposes. Denote $w_{a j}=$ $-\ln (p(a \rightarrow j))$ for $a \in A$ and $j \in P_{a}$, and let $w(f)=\sum_{a \in A} w_{a f(a)}$. It is easy to see that an optimal assignment $f$ can be found by minimizing $w(f)$, which is linear. The problem is defined as follows:

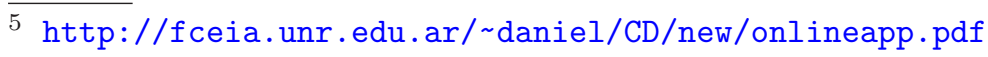


$\frac{\mathscr{K} \text {-Matching Problem }}{I N S T A N C E:}$

$n_{A}, n_{B} \in \mathbb{Z}_{+}$

$A, B$ such that $|A|=n_{A},|B|=n_{B}$;

$P_{a} \subset \mathscr{P}(B)$ such that $|j| \leq \mathscr{K}$ for all $j \in P_{a}$, for all $a \in A$;

$w_{a j} \in \mathbb{R}_{+}$for all $j \in P_{a}$ such that $\sum_{j \in P_{a}} e^{-w_{a j}}=1$, for all $a \in A$.

OBJECTIVE: Obtain a valid assigment $f$ such that $w(f)$ is minimum.

Below, I show that this problem can be polynomially transformed to the MWSSP. Recall that, given a graph $G=(V, E)$ and weights $z \in \mathbb{R}_{+}^{V}$, MWSPP consists of finding a stable set $S \subset V$ of $G$ such that $z(S)=\sum_{v \in S} z_{v}$ is maximum. Let $G=(V, E)$ be the graph such that $V=\left\{v_{a j}: a \in A, j \in P_{a}\right\}$,

$$
\begin{aligned}
& E=\left\{\left(v_{a j}, v_{a j^{\prime}}\right): a \in A, \quad j, j^{\prime} \in P_{a}, \quad j \neq j^{\prime}\right\} \cup \\
& \left\{\left(v_{a j}, v_{a^{\prime} j^{\prime}}\right): a, a^{\prime} \in A, \quad a \neq a^{\prime}, \quad j \in P_{a}, \quad j^{\prime} \in P_{a^{\prime}}, \quad j \cap j^{\prime} \neq \emptyset\right\},
\end{aligned}
$$

and consider weights $z_{a j}=M-w_{a j}$ where $M=\sum_{a \in V} \sum_{j \in P_{a}} w_{a j}$.

Theorem 2.1 Let $S$ be an optimal stable set of the MWSSP. The $\mathscr{K}$-Matching Problem is feasible if and only if $z(S)>M .\left(n_{A}-1\right)$ and, in that case, $f(a)=j$ for all $v_{a j} \in S$ is an optimal assignment of the $\mathscr{K}$-Matching Problem.

Proof. If the $\mathscr{K}$-Matching Problem is feasible, there exists a valid assignment $\hat{f}$. Let $\hat{S} \subset V$ such that $v_{a j} \in \hat{S}$ if and only if $\hat{f}(a)=j$. It is easy to see that $\hat{S}$ is a stable set of $G$ whose weight is greater than M. $\left(n_{A}-1\right)$. Since $S$ is optimal, $z(S) \geq z(\hat{S})>M .\left(n_{A}-1\right)$.

Conversely, assume that $z(S)>M .\left(n_{A}-1\right)$ and let $f(a)=j$ for all $v_{a j} \in S$. First, let us prove that $f$ is a valid assignment. Suppose that there exists $a^{*} \in A$ such that $v_{a^{*} j} \notin S$ for every $j$. Then, $z(S) \leq M .\left(n_{A}-1\right)-\sum_{v_{a j} \in S} w_{a j} \leq$ $M$. $\left(n_{A}-1\right)$ which leads to a contradiction. Then, $f$ is defined for all $a \in A$. In addition, if $v_{a j}, v_{a j^{\prime}} \in S$ then $v_{a j}=v_{a j^{\prime}}$ so $a$ is assigned to a unique $j$. Furthermore, if $a, a^{\prime} \in A$ and $b \in B$ such that $b \in j$ and $b \in j^{\prime}$ for some $j \in P_{a}, j^{\prime} \in P_{a^{\prime}}$ then $a=a^{\prime}$ so $b$ is assigned to at most one star of $A$. Now, let us prove that $f$ is optimal. Suppose that there exists a valid assignment $\hat{f}$ such that $w(\hat{f})<w(f)$. Again, let $\hat{S} \subset V$ such that $v_{a j} \in \hat{S}$ if and only if $\hat{f}(a)=j$. It is easy to see that $\hat{S}$ is a stable set of $G$ whose weight is $M . n_{A}-w(\hat{f})$. Then, $z(\hat{S})>M . n_{A}-w(f)=z(S)$, which is absurd.

Based on this reduction, an exact algorithm (which can be consulted in the Online Appendix) was implemented for solving instances of the 2-Matching Problem. Then, a real catalog of 52313 stars (where 568 are doubles) was 


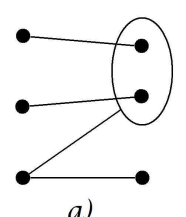

a)

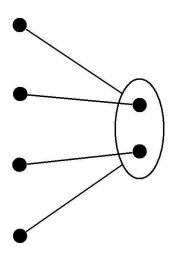

6)

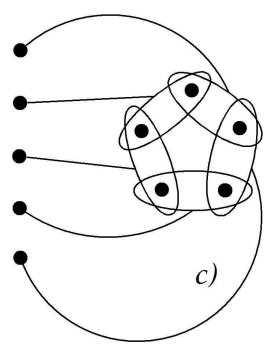

Fig. 1. Instances for: a) claw, b) diamond, c) odd hole $C_{5}$

cross-identified against another of 83397 stars in less than a minute of CPU time. The algorithm, auxiliary files and the resulting catalog are available [3].

Now, define $\mathcal{F}_{\mathscr{K}}$ as the family of graphs $G$ obtained by the previous reduction for any instance of the $\mathscr{K}$-Matching Problem. It is clearly that the 1-Matching Problem, i.e. when no multiple stars are present in catalog $A$, can be trivially reduced to the classic Maximum Weighted Matching Problem (MWMP) over a bipartite graph $G_{B}$. Indeed, our reduction gives the line graph of $G_{B}$. Therefore, $\mathcal{F}_{1}$ is the family of line graphs of bipartite graphs. It is known from Graph Theory that, if $G$ belongs to such family, then the claw, the diamond and the odd holes are forbidden induced subgraphs of $G$. This leads to the following:

Open question. Which are the forbidden induced subgraphs that characterize those graphs from $\mathcal{F}_{\mathscr{K}}$ for $\mathscr{K} \geq 2$ ?

Although none of the mentioned subgraphs are forbidden for the case $\mathscr{K} \geq$ 2 (they can be generated from instances of the 2-Matching Problem as it is shown in Figure 1), the claw can be generalized as follows:

Lemma 2.2 For $\mathscr{K} \geq 1$, let $G \in \mathcal{F}_{\mathscr{K}}$. Then, $G$ is $K_{1, \mathscr{K}+2}$-free.

Proof. Suppose that the star $K_{1, \mathscr{K}+2}$ is an induced subgraph of $G$. Let $v_{a j}$ be the central vertex of the star and $v_{a_{1}, j_{1}}, v_{a_{2}, j_{2}}, \ldots, v_{a_{\mathscr{K}+2}, j_{K}+2}$ the remaining vertices. W.l.o.g., we can assume that $a \neq a_{1}, a \neq a_{2}, \ldots, a \neq a_{r}, a=$ $a_{r+1}=a_{r+2}=\ldots=a_{\mathscr{K}+2}$ for some $r$. If $r \leq \mathscr{K}$, we would obtain that $a=a_{\mathscr{K}+1}=a_{\mathscr{K}+2}$ and then $v_{a, j_{\mathscr{K}+1}}$ and $v_{a, j_{\mathscr{K}+2}}$ would be adjacent which is absurd. Therefore, $r \geq \mathscr{K}+1$. Since $v_{a j}$ and $v_{a_{i}, j_{i}}$ are adjacent and $a \neq a_{i}$ for all $1 \leq i \leq \mathscr{K}+1$, then $j \cap j_{i} \neq \emptyset$. On the other hand, $v_{a_{i}, j_{i}}$ and $v_{a_{i^{\prime}}, j_{i^{\prime}}}$ are not adjacent for all $1 \leq i<i^{\prime} \leq \mathscr{K}+1$, then $j_{i} \cap j_{i^{\prime}}=\emptyset$. Therefore, $j$ should have at least $\mathscr{K}+1$ elements which leads to a contradiction.

Another forbidden subgraph of the 2-Matching Problem is given as follows. Let $G$ be the graph of Figure 2(a). Note that the instance of the 2-Matching 

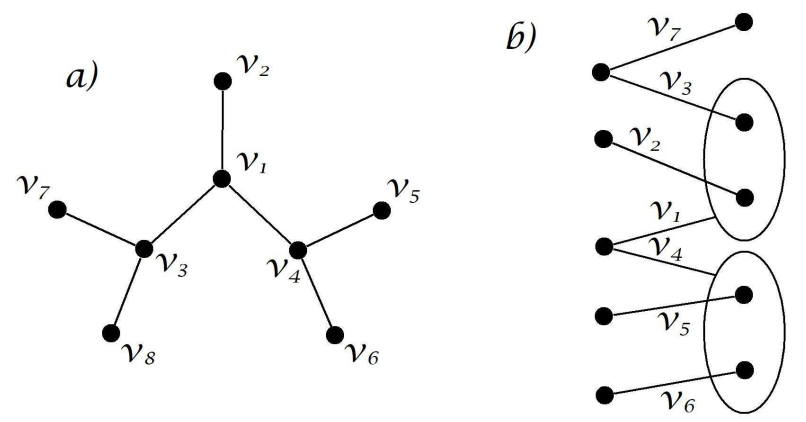

Fig. 2. A graph not in $\mathcal{F}_{2}$ : a) $G$, b) partial construction

Problem given in Figure 2(b) corresponds to the subgraph of $G$ induced by vertices $v_{1}, \ldots, v_{7}$. A drawback emerges when $v_{8}$ is considered. Hence, $G \notin \mathcal{F}_{2}$.

From the complexity point of view, the $\mathscr{K}$-Matching Problem for $\mathscr{K}=1$ is polynomial due to the existence of efficient algorithms for the MWMP such as the Hungarian Algorithm. When $\mathscr{K}=2$, Lemma 2.2 says that graphs from $\mathcal{F}_{\mathscr{K}}$ are $K_{1,4}$-free, and the MWSSP for $K_{1,4}$-free graphs is known to be $\mathcal{N P}$-Hard. Nevertheless, this does not mean that our matching problem is hard since $\mathcal{F}_{2}$ has other forbidden subgraphs. Its complexity is addressed in the next section.

\section{Complexity of the problem}

In this section, I prove that the $\mathscr{K}$-Matching Problem is $\mathcal{N} \mathcal{P}$-hard for $\mathscr{K} \geq 2$. Even more, I consider a more restricted problem where every star of $A$ has exactly multiplicity $\mathscr{K}$. The decision problem is as follows:

$\mathscr{K}$-Matching Decision Problem $(\mathscr{K}$-MDP $)$

INSTANCE: $n_{A}, n_{B} \in \mathbb{Z}_{+} ; A, B$ such that $|A|=n_{A},|B|=n_{B} ; \quad P_{a} \subset \mathscr{P}(B)$ such that $|j|=\mathscr{K}$ for all $j \in P_{a}$, for all $a \in A ; w_{a j} \in \mathbb{R}_{+}$for all $j \in P_{a}$ such that $\sum_{j \in P_{a}} e^{-w_{a j}}=1$, for all $a \in A ; t \in \mathbb{R}$.

QUESTION: Is there a valid assignment $f$ such that $w(f) \leq t$ ?

Let us first introduce two auxiliary problems. Given $n \in \mathbb{Z}_{+}$, let $\mathcal{P}$ and $\mathcal{Q}$ be disjoint sets such that $|\mathcal{P}|=|\mathcal{Q}|=n$. A perfect matching (p.m. for short) is a set $M \subset \mathcal{P} \times \mathcal{Q}$ such that $|M|=n$ and every element of $\mathcal{P} \cup \mathcal{Q}$ occurs in exactly one pair of $M$. The first, which is $\mathcal{N} \mathcal{P}$-complete [4], is defined below:

Disjoint Matchings (DM)

$\overline{I N S T A N C E: ~} n \in \mathbb{Z}_{+} ;$disjoint sets $\mathcal{P}, \mathcal{Q}$ such that $|\mathcal{P}|=|\mathcal{Q}|=n ; \mathcal{A}_{1}, \mathcal{A}_{2} \subset \mathcal{P} \times \mathcal{Q}$. QUESTION: Are there p.m. $M_{1} \subset \mathcal{A}_{1}, M_{2} \subset \mathcal{A}_{2}$ such that $M_{1} \cap M_{2}=\emptyset$ ? 
The second auxiliary problem is given below. It differs from the 2-Matching Decision Problem in that values $w_{a j}$ do not come from probabilities:

2-Matching Decision Problem with Arbitrary Weights (2-MDPAW)

INSTANCE: $n_{A}, n_{B} \in \mathbb{Z}_{+} ; \quad$ sets $A, B$ such that $|A|=n_{A}$ and $|B|=n_{B} ; \quad P_{a} \subset$ $\mathscr{P}(B)$ such that $|j|=2$ for all $j \in P_{a}, a \in A ; w_{a j} \in \mathbb{R}_{+}$for all $j \in P_{a}, a \in A ; t \in \mathbb{R}$. QUESTION: Is there a valid assignment $f$ such that $w(f) \leq t$ ?

Lemma 3.1 2-MDPAW is $\mathcal{N} \mathcal{P}$-complete.

Proof. First of all, it clearly is $\mathcal{N} \mathcal{P}$. Below, a polynomial transformation from $\mathrm{DM}$ is proposed. Consider an instance $\mathcal{P}=\left\{p_{1}, \ldots, p_{n}\right\}, \mathcal{Q}=\left\{q_{1}, \ldots, q_{n}\right\}$, $\mathcal{A}_{1}, \mathcal{A}_{2} \subset \mathcal{P} \times \mathcal{Q}$ of DM. We construct an instance of 2-MDPAW as follows. Let $A=\left\{a_{r s}: r\right.$ and $s$ such that $\left.\left(p_{r}, q_{s}\right) \in \mathcal{A}_{1} \cup \mathcal{A}_{2}\right\}$ and

$$
\begin{aligned}
& B=\left\{p_{i}^{1}, p_{i}^{2}: i \text { such that } p_{i} \in \mathcal{P}\right\} \cup \\
& \qquad\left\{q_{i}^{1}, q_{i}^{2}: i \text { such that } q_{i} \in \mathcal{Q}\right\} \cup \\
& \qquad\left\{z_{r s}, z_{r s}^{\prime}: r \text { and } s \text { such that } a_{r s} \in A\right\} .
\end{aligned}
$$

Hence, $n_{A}=\left|\mathcal{A}_{1} \cup \mathcal{A}_{2}\right|$ and $n_{B}=4 n+2\left|\mathcal{A}_{1} \cup \mathcal{A}_{2}\right|$. For every $a_{r s} \in A$, let $P_{a_{r s}}=\left\{\left\{p_{r}^{i}, q_{s}^{i}\right\}: r, s\right.$ and $i$ such that $\left.\left(p_{r}, q_{s}\right) \in \mathcal{A}_{i}\right\} \cup\left\{\left\{z_{r s}, z_{r s}^{\prime}\right\}\right\}$. For $a_{r s} \in A$ and $j \in P_{a_{r s}}$, let

$$
w_{a_{r s} j}= \begin{cases}0, & j=\left\{p_{r}^{i}, q_{s}^{i}\right\} \text { for some } i \wedge\left(p_{r}, q_{s}\right) \in \mathcal{A}_{1} \triangle \mathcal{A}_{2}, \\ 1, & j=\left\{p_{r}^{i}, q_{s}^{i}\right\} \text { for some } i \wedge\left(p_{r}, q_{s}\right) \in \mathcal{A}_{1} \cap \mathcal{A}_{2}, \\ 1, & j=\left\{z_{r s}, z_{r s}^{\prime}\right\} \wedge\left(p_{r}, q_{s}\right) \in \mathcal{A}_{1} \triangle \mathcal{A}_{2}, \\ 2, & j=\left\{z_{r s}, z_{r s}^{\prime}\right\} \wedge\left(p_{r}, q_{s}\right) \in \mathcal{A}_{1} \cap \mathcal{A}_{2},\end{cases}
$$

where $\triangle$ denotes the symmetric difference operator between sets. Finally, let $t=\left|\mathcal{A}_{1}\right|+\left|\mathcal{A}_{2}\right|-2 n$.

We prove that, given disjoint p.m. $M_{1} \subset \mathcal{A}_{1}, M_{2} \subset \mathcal{A}_{2}$, there exists a valid assignment $f$ such that $w(f) \leq t$. Consider $f\left(a_{r s}\right)=\left\{p_{r}^{i}, q_{s}^{i}\right\}$ when $\left(p_{r}, q_{s}\right) \in M_{i}$ for some $i \in\{1,2\}$, and $f\left(a_{r s}\right)=\left\{z_{r s}, z_{r s}^{\prime}\right\}$ otherwise. The validity of $f$ is straightforward. Also, $w(f)=\left|\left(M_{1} \cap \mathcal{A}_{2}\right) \cup\left(M_{2} \cap \mathcal{A}_{1}\right)\right|+$ $\left|\left(\mathcal{A}_{1} \backslash\left(M_{1} \cup \mathcal{A}_{2}\right)\right) \cup\left(\mathcal{A}_{2} \backslash\left(M_{2} \cup \mathcal{A}_{1}\right)\right)\right|+2\left|\left(\mathcal{A}_{1} \cap \mathcal{A}_{2}\right) \backslash\left(M_{1} \cup M_{2}\right)\right|=\left|\mathcal{A}_{1} \backslash M_{1}\right|+$ $\left|\mathcal{A}_{2} \backslash M_{2}\right|=t$. Conversely, we prove that, for a given valid assignment $f$ such that $w(f) \leq t$, there exist disjoint p.m. $M_{1} \subset \mathcal{A}_{1}, M_{2} \subset \mathcal{A}_{2}$. Consider $M_{i}=$ $\left\{\left(p_{r}, q_{s}\right): r\right.$ and $s$ such that $\left.f\left(a_{r s}\right)=\left\{p_{r}^{i}, q_{s}^{i}\right\}\right\}$ for all $i \in\{1,2\}$. Since $f$ is a function, $M_{1} \cap M_{2}=\emptyset$. It is also straightforward that $M_{i} \subset \mathcal{A}_{i}$. Now, suppose that there exists an element in $\mathcal{P} \cup \mathcal{Q}$ occurring in two pairs of $M_{i}$. W.l.o.g., 
suppose $\left(p_{1}, q_{1}\right),\left(p_{1}, q_{2}\right) \in M_{1}$. Then, $f\left(a_{11}\right) \cap f\left(a_{12}\right)=\left\{p_{1}^{1}, q_{1}^{1}\right\} \cap\left\{p_{1}^{1}, q_{2}^{1}\right\} \neq \emptyset$ which is absurd. Therefore, every element in $\mathcal{P} \cup \mathcal{Q}$ occur at most once in any pair of $M_{1}$ and once in $M_{2}$. It is easy to see that $\left|M_{1}\right| \leq n$ and $\left|M_{2}\right| \leq n$. Suppose that there exists an element in $\mathcal{P} \cup \mathcal{Q}$ which does not occur in any pair of $M_{i}$. Again, w.l.o.g., suppose that such element does not occur in $M_{1}$. Then, $\left|M_{1}\right|<n$ and $w(f)=\left|\mathcal{A}_{1} \backslash M_{1}\right|+\left|\mathcal{A}_{2} \backslash M_{2}\right|>\left|\mathcal{A}_{1}\right|+\left|\mathcal{A}_{2}\right|-2 n=t$. Absurd! Therefore, $M_{1}$ and $M_{2}$ are both p.m. and $\left|M_{1}\right|=\left|M_{2}\right|=n$.

Theorem $3.2 \mathscr{K}$-MDP is $\mathcal{N} \mathcal{P}$-complete for all $\mathscr{K} \geq 2$.

Proof. We propose a polynomial transformation from 2-MDPAW. Consider an instance $A=\left\{a_{1}, \ldots, a_{n_{A}}\right\}, B=\left\{b_{1}, \ldots, b_{n_{B}}\right\}, P_{a}, w_{a j}$, and $t$ of 2-MDPAW. We construct an instance $A^{\prime}, B^{\prime}, P_{a}^{\prime}, w_{a j}^{\prime}, t^{\prime}$ of $\mathscr{K}$-MDP as follows. Let $A^{\prime}=$ $A \cup\left\{\bar{a}_{1}, \ldots, \bar{a}_{n_{A}}\right\}$ and $B^{\prime}=B \cup\left\{\tilde{b}_{j k}: j \in \bigcup_{a \in A} P_{a}, 3 \leq k \leq \mathscr{K}\right\} \cup\left\{\bar{b}_{a k}: a \in\right.$ $A, 1 \leq k \leq \mathscr{K}\}$. For all $a \in A$, let $P_{a}^{\prime}=\left\{j \cup \bigcup_{k=3}^{\mathscr{K}} \tilde{b}_{j k}: j \in P_{a}\right\} \cup\left\{j_{a}^{\prime}\right\}$ where $j_{a}^{\prime}=\bigcup_{k=1}^{\mathscr{K}} \bar{b}_{a k}$ (if $\mathscr{K}=2$, we just have $\left.P_{a}^{\prime}=P_{a} \cup\left\{\left\{\bar{b}_{a 1}, \bar{b}_{a 2}\right\}\right\}\right)$. Take an $a^{*} \in A$ that maximizes $p^{*} \doteq \sum_{j \in P_{a^{*}}} e^{-w_{a^{*} j}}$. Let $\beta>\ln \left(p^{*}\right)$ and $w_{a j}^{\prime}=w_{a j}+\beta$ for all $j \in P_{a}, a \in A$. Then, $\sum_{j \in P_{a}} e^{-w_{a j}^{\prime}}<1$. Let $w_{a j_{a}^{\prime}}^{\prime}=-\ln \left(1-\sum_{j \in P_{a}} e^{-w_{a j}^{\prime}}\right)$ for all $a \in A$. We obtain $\sum_{j \in P_{a}^{\prime}} e^{-w_{a j}^{\prime}}=1$. For all $i \in\left\{1, \ldots, n_{A}\right\}$, let $P_{\bar{a}_{i}}^{\prime}=\left\{j_{\bar{a}_{i}}^{\prime}\right\}$ and $w_{\bar{a}_{i} j_{\bar{a}_{i}}^{\prime}}^{\prime}=0$ where $j_{\bar{a}_{i}}^{\prime}=\bigcup_{k=1}^{\mathscr{K}} \bar{b}_{a_{i} k}$. Finally, let $t^{\prime}=t+n_{A} \beta$.

Now we prove that there is an $f$ of 2 -MDPAW such that $w(f) \leq t$ if and only if there is an $f^{\prime}$ of $\mathscr{K}$-MDP such that $w\left(f^{\prime}\right) \leq t^{\prime}$. In order $f^{\prime}$ to be valid, $f^{\prime}\left(\bar{a}_{i}\right)=j_{\bar{a}_{i}}^{\prime}$ for all $1 \leq i \leq n_{A}$. We propose $f^{\prime}(a)=f(a)$ for all $a \in A$. Clearly, if $f$ is valid then $f^{\prime}$ is valid too, and conversely. Since $\sum_{a \in A^{\prime} \backslash A} w_{a f^{\prime}(a)}^{\prime}=0$, $w\left(f^{\prime}\right)=w(f)+n_{A} \beta$.

\section{References}

[1] Budavári T. and A. Basu, Probabilistic Cross-Identification in Crowded Fields as an Assignment Problem, Astron. J. 152 (2016), 86B.

[2] Severín D. E., and D. J. Sevilla, Development of a new digital version of "Cordoba Durchmusterung" stellar catalog, Revista Académica Electrónica de la U.N.R., 1 (2015), 2250-2260.

[3] Severín D. E., Cross-identification between Cordoba Durchmusterung catalog (declinations -22, -23 and -24) and PPMX catalog, Mendeley Data, v1 (2018). http://dx.doi.org/10.17632/5wwwtv7c8c.1

[4] Frieze A. M., Complexity of a 3-dimensional assignment problem, Eur. J. Oper. Res. 13 (1983), 161-164. 